DOI: $10.17725 /$ rensit.2019.11.351

\title{
Modeling vertical fractures using the Schoenberg model on structured grids by grid-characteristic method
}

\section{Polina V. Stognii}

Educational Scientific and Experimental Center of Moscow Institute of Physics and Technology, http://kmipt.ru/

Dolgoprudny 141700, Moscow region, Russian Federation

E-mail:stognii@phystech.edu

Nikolay I. Khokhlov, Igor B. Petrov

Scientific Research Institute for System Analysis of Russian Academy of Sciences, https://www.niisi.ru/

Moscow 117218, Russian Federation

E-mail:k_h@inbox.ru,petrov@mipt.ru

Received 28.08.2019, peer reviewed 16.09.2019, accepted 30.09.2019

Abstract. Seismic prospecting is one of the most popular methods of revealing oil and gas reservoirs in the North seas. Geological media, which contain hydrocarbon layers, often include different heterogeneities, for example, fractures. Many scientific works are devoted to modelling fractures and ways of considering them in various mathematical problems, but the Linear Slip model, known as Schoenberg model of fracture, is still the most actual, though this model was developed at the end of the last century. This model is characterized by the presence of an additional parameter so called fracture opening parameter, describing fractures. In this work, we present the algorithm of introducing the Schoenberg fracture model in the grid-characteristic method. The fracture is organized to be parallel to the boundaries of the modelling grid. The fracture is situated on the border of the two media with the same characterizing parameters. We describe in detail the algorithm of computing the meanings of velocity and stress tensor in the points on the border with the fracture. In order to verify the correctness of the developed approach to modelling a fracture using the Schoeberg fracture model, we carry out the test calculations of sesmic waves spread in a homogeneous media with the vertical extremely thin fracture, described by the not equal to null normal component of the fracture opening coefficient and with the equal to null tangential component of the fracture opening coefficient.

Keywords: seismic prospecting, numerical modelling, grid-characteristic method, fractured media UDC 519.63

Acknowledgments: This work was financially supported by the Russian Federal Property Fund in the framework of scientific project No. 19-01-00281.

For citation: Polina V. Stognii, Nikolay I. Khokhlov, Igor B. Petrov. Modeling vertical fractures using the Schoenberg model on structured grids by grid-characteristic method. RENSIT, 2019, 11(3):351-356; DOI: 10.17725/rensit.2019.11.351.

Contents

1. INTRODUCTION (351)

2. Determinant equations (352)

3. Numerical method (352)

4. Results (354)

5. Conclusion (355)

REFERENCES (355)

\section{INTRODUCTION}

Fractures are often a barrier on the way of exploring the chosen territory during conducting the geological works on oil and gas deposits extraction $[1,2]$. They bring in a significant summary into the seismogramms, got as a result of geological layers exploration. Therefore, it is necessary to take into account fractured media while modelling the chosen area in order to obtain more accurate results of seismic data processing.

In reality, the sizes of most fractures are of that sort that the ratio of their width to their height is equal to zero in the limit [3]. These fractures are described by an abstract model of an extremely thin 
fracture. In this work, we explore only models of extremely thin fractures. A fracture is described by a set of contact and border conditions in the place of its establishment in the computational area.

There are different ways of modelling the seismic waves spread in fractured media. For example, hierarhical grids are applied to modelling statical fractures [4]. In the work [5], authors use the pseudospectral method for modelling fractures with not equal to zero parameter of viscosity.

Hudson model [6] and Schoenberg model [7] are the most well-known models, used for describing fractures. In the Hudson model, a fracture is described by the parameter of effective stiffness, which is linearly or quadratic (first or second order of accuracy, accordingly) dependent from the socalled parameter of fracture density [8].

In Schoenberg model $[9,10]$, the condition of equal normal components of the stress tensor is set on the boundary of a fracture. In addition, the way of computing the normal components through special parameters of fracture opening, dependent from the medium, filling the fracture, is supposed. The Schoenberg model was already introduced in the well-known Galerkin method, the results can be found in [11].

In this work, we introduce the method of computing geological media with a fracture with the help of the grid-characteristic method and the induced Schoenberg model for the $2 D$ case, which is done for this method for the first time. The contact conditions on the boundary with the fracture are described in detail. In addition, we present the example of numerical modelling for the seismic reflection from the fracture on the boundary of two media.

\section{DETERMINANT EQUATIONS}

For describing the spread of elastic waves in a homogeneous medium, we used the system of linear-elastic equations [12]:

$$
\begin{aligned}
& \rho \frac{\partial}{\partial t} v=(\nabla \cdot \sigma)^{T}, \\
& \frac{\partial}{\partial t} \sigma=\lambda(\nabla \cdot v) I+\mu\left((\nabla \times v)+(\nabla \times v)^{T}\right),
\end{aligned}
$$

where $\rho$ is the medium density, $v$ is the speed of elastic waves spread in the medium, $\sigma$ is the Caushy stress tensor, $\lambda$ and $\mu$ are the Lame parameters, determining the properties of the elastic material.

For fracture describing, we used the equations from the model of Schoenberg [13]:

$$
\begin{aligned}
& \sigma_{x x}^{\text {left }}=\sigma_{x x}^{\text {right }}, \\
& \sigma_{x y}^{\text {left }}=\sigma_{x y}^{r i g h t}, \\
& \frac{\partial \sigma_{x x}}{\partial t}=K_{N}\left(v_{x}^{\text {right }}-v_{x}^{\text {left }}\right), \\
& \frac{\partial \sigma_{x y}}{\partial t}=K_{T}\left(v_{y}^{r i g h t}-v_{y}^{l e f t}\right) .
\end{aligned}
$$

In (3)-(6) the indexes «left» and «right» denote the medium on the one side (left) and the medium on the other side (right) from the fracture.

$K_{N}$ and $K_{T}$ are the normal and tangential components of the parameter of fracture opening. These characteristics are known beforehand or can be calculated. The description of the theoretical computation of normal and tangential components of fracture opening are presented in the work [14], the description of calculating the parameter of fracture opening as a result of the laboratory experiment is presented in the work [15]. The width of the fracture is considered to be extremely small.

\section{NUMERICAL METHOD}

For solving the system of linear-elastic equations (1), (2) we used the grid-characteristic method [16]. For computing the homogeneous medium, we applied the Laks-Wendroff scheme of the second order of accuracy [17] :

$$
\begin{aligned}
& u_{i}^{n+1}=u_{i}^{n}-\frac{c t}{2 h}\left(u_{i+1}^{n}-u_{i-1}^{n}\right)+ \\
& +\frac{c^{2} t^{2}}{2 h^{2}}\left(u_{i+1}^{n}+u_{i-1}^{n}-2 u_{i}^{n}\right) .
\end{aligned}
$$

In order to compute fractures, we also used the grid-characteristic method, but with some modifications. Now, we examine the algorithm of computing points on the boundary with the fracture in more detail.

For this, we present the system of equations (1), (2) in the following form: 


$$
\frac{\partial q}{\partial t}+A_{x} \frac{\partial q}{\partial x}+A_{y} \frac{\partial q}{\partial y}=0
$$

where $q=\left\{\sigma_{x x}, \sigma_{x y}, \sigma_{y y}, v_{x}, v_{y}\right\} . A_{x}$ and $A_{y}$ are matrixes, made up from the corresponding components of the equations (1), (2):

$$
\begin{aligned}
& A_{x}=\left(\begin{array}{ccrrr}
0 & 0 & -\frac{1}{p} & 0 & 0 \\
0 & 0 & 0 & 0 & -\frac{1}{p} \\
-\lambda-2 \mu & 0 & 0 & 0 & 0 \\
0 & -\lambda-2 \mu & 0 & 0 & 0 \\
0 & -\mu & 0 & 0 & 0
\end{array}\right) \\
& A_{y}=\left(\begin{array}{ccccc}
0 & 0 & 0 & 0 & -\frac{1}{p} \\
0 & 0 & 0 & -\frac{1}{p} & 0 \\
0 & -\lambda & 0 & 0 & 0 \\
-\lambda & 0 & 0 & 0 & 0 \\
-\mu & 0 & 0 & 0 & 0
\end{array}\right) .
\end{aligned}
$$

As a result of adopting the method of coordinates splitting in space, we obtain two $1 D$ systems of equations:

$$
\frac{\partial q}{\partial t}+A_{i} \frac{\partial q}{\partial i}=0, \quad i=x, y \text {. }
$$

Now, we examine the system of equations (11) for the $x$-coordinate:

$$
\frac{\partial q}{\partial t}+A_{x} \frac{\partial q}{\partial x}=0
$$

Hereinafter, we consider the fracture, situated perpendicular to the $X$-axis, parallel to the boundaries of the computational grid cells. We can make analogous transformations, which are presented lower, for the case, when the fracture is located perpendicular to the $Y$-axis.

The system of equations (12) is hyperbolic, therefore it can be represented in the form :

$$
\frac{\partial q}{\partial t}+\Omega_{x} \Lambda_{x} \Omega_{x}^{-1} \frac{\partial q}{\partial x}=0
$$

where $\Omega_{\mathrm{x}}$ is the matrix, made of the eigenvectors, $\Lambda_{\mathrm{x}}$ is the matrix with the eigenvalues on the diagonal.

The eigenvalues of the matrixes $A_{x}, A_{y}$ are: $\{-c$, $\left.c_{p},-c_{s}, c_{s}, 0\right\}$, where $c_{p}$ is the longitudinal sound velocity, $c_{s}$ is the transverse sound velocity.

After the variable change, the system (13) will look as following:

$$
\frac{\partial p}{\partial t}+\Lambda_{x} \frac{\partial p}{\partial x}=0
$$

The system (14) contains five independent equations, which can be solved by any differential scheme [17].

We examine the case of computing points on the boundary of the fracture with the help of the CIR scheme [17]. The scheme looks as follows:

$$
\begin{aligned}
& u_{i}^{n+1}=u_{i}^{n}-\frac{c t}{h}\left(u_{i+1}^{n}-u_{i}^{n}\right), \text { if } c>0, \\
& u_{i}^{n+1}=u_{i}^{n}-\frac{c t}{h}\left(u_{i-1}^{n}-u_{i}^{n}\right), \text { if } c<0 .
\end{aligned}
$$

In (15), (16) the index $i$ signifies the number of coordinate (for example, $x$ ), the index $n$ denotes the number of time, $t$ is the time step, $b-$ is the coordinate step, $c$ is velocity. From equations (15), (16), it follows that points on the left side of the fracture can be calculated only for vectors, corresponding to negative eigenvalues $(-c,-c)$. Points on the right side of the fracture can be calculated only for vectors, corresponding to positive eigenvalues $\left(c_{p}, c_{s}\right)$. For solving the remaining two systems of equations on the left and on the right, we need additional boundary conditions (3)-(6).

Now, we explore in detail the algorithm of computing points on the left side of the fracture for positive characteristics (the algorithm of computing points on the right side of the fracture for negative characteristics is the same). For this, we introduce an additional ghost-node border+1 on the right side of the border node [18] into the examined computational grid and place such values into the node border +1 , so that the conditions (3), (4) are fulfilled on the boundary of the fracture on the next time step. The conditions (5), (6) allow to calculate the values $\sigma_{x x}, \sigma_{x y}$ on the $(n+1)$-th time step. In this work, we solved 
the equations (5), (6) with the help of the Eiler method:

$$
\begin{aligned}
& \sigma_{x x}^{\text {left }, n+1}=\sigma_{x x}^{l e f t, n}+K_{N}\left(v_{x}^{\text {right }, n}-v_{x}^{\text {left }, n}\right), \\
& \sigma_{x y}^{l e f t, n+1}=\sigma_{x y}^{l e f t, n}+K_{T}\left(v_{y}^{r i g h t, n}-v_{y}^{l e f t, n}\right) .
\end{aligned}
$$

For describing the boundary conditions, we used the non-reflecting boundary conditions [19]:

$$
\Omega^{\text {out }} u=0 \text {, }
$$

where $\Omega^{\text {out }}$ is the matrix of the eigenvectors, corresponding to the outcoming characteristics (in other words, negative meanings of the characteristics for the matrixes $A_{2}, A_{j}$ ).

\section{RESULTS}

We carried out the numerical modelling of the seismic response from an extremely long fracture in a homogeneous medium for the $2 D$ formulation of the problem. The initial impulse was described through the definition of the stress tensor inside a unit circle with the help of the following formula:

$$
\begin{aligned}
& \sigma_{x x}(x, y)=\sigma_{y y}(x, y)= \\
& =\exp \left(\frac{-(x-2.2)^{2}}{0.035}-\frac{(y-3.0)^{2}}{0.035}\right), \\
& 1.7<x<2.7,2.5<y<3.5 .
\end{aligned}
$$

The homogeneous medium was described by the following parameters. The speed of the longitudinal waves was equal to $2 \mathrm{~m} / \mathrm{sec}$., the speed of the transverse waves $-1 \mathrm{~m} / \mathrm{sec}$. The medium density was equal to $1 \mathrm{~kg} / \mathrm{m}^{3}$. The computational area was $9 \mathrm{~m}$ along the $\mathrm{X}$-axis and $6 \mathrm{~m}$ along the Y-axis. The extremely thin fracture along the whole computational grid was situated vertically on the boundary $x=3 \mathrm{~m}$ (Fig. 1).

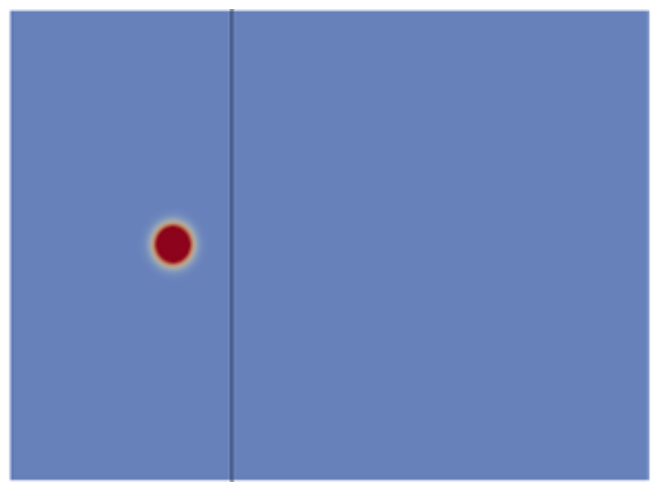

Fig. 1. Wave patterns for the seismic impulse spread (namely, the normal component of the stress tensor $\sigma_{x x}$ ) in the homogeneous medium at different consistent moments of time.

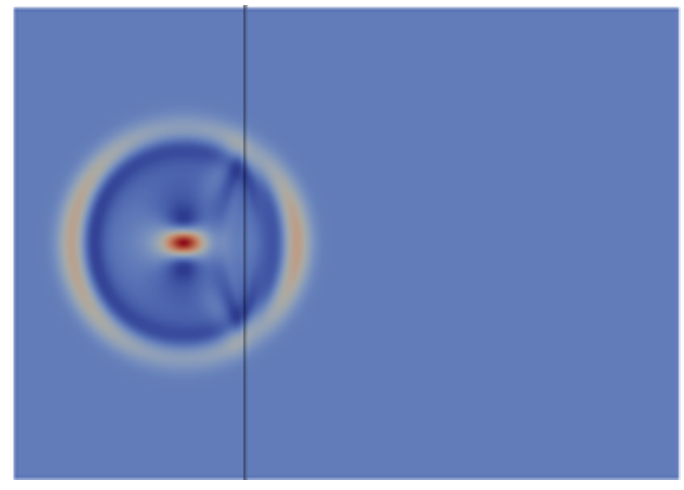

Fig. 2. The seismic impulse at time 0.7 sec.

In the calculations, the time step was equal to 0.005 sec., the coordinate step for $X$ - and $Y$-axis was $0.025 \mathrm{~m}$. The value of the normal component of the fracture opening parameter $K_{N}$ was equal to $400 \mathrm{~Pa} / \mathrm{m}$, the value of the tangential component of the fracture opening parameter $K_{T}$ was $0 \mathrm{~Pa} / \mathrm{m}$. The same medium with the parameters, described earlier in this section, is situated on the left and on the right side of the fracture. We calculated the spreads of the seismic impulse in the right and left areas relative to the fracture independently, excepting the border points, where we changed the values of the normal and tangent components of the stress tensor (according to the border conditions (3)-(6)). At this stage, we made all the calculations one after the other. Later on, the calculations will become faster with the help of parallelization, for example, using the MPI technology [20].

Figures 1, 2, 3 depict wave patterns of the seismic impulse spread (namely, the normal component of the stress tensor $\sigma_{x x}$ ). Fig. 1 presents the seismic impulse at the initial moment of time. Figs 2, 3 introduce the consistent momemts of the seismic wave reflection from the fracture and its further spread in the homogeneous medium .

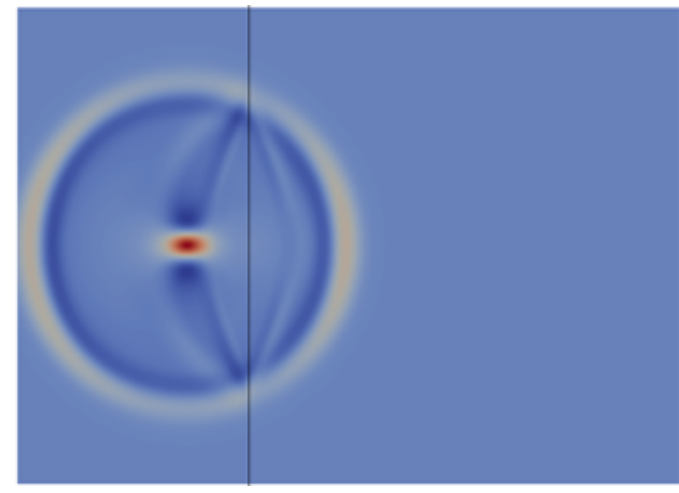

Fig. 3. The seismic impulse at time $1.0 \mathrm{sec}$. 


\section{CONCLUSION}

In this work, we introduced the realization of the Schoenberg fracture model into the gridcharacteristic method. Weinterposed the Schoenberg model, which is widely used for modelling fractured media, in the grid-characteristic method with the help of the additional corresponding boundary conditions in the computational area, where the fracture is situated. We presented the detailed description of calculating the points on the boundary of the fracture for the $2 D$ case. The boundary conditions were introduced through the CIR scheme of the first order of accuracy, while the other points of the computational grid were calculated using the Laks-Wendroff scheme of the second order of accuracy.

As an example, we carried out the test calculation of the seismic waves spread in the homogeneous medium with the vertical fracture for the case, when the tangential component of the fracture opening parameter was equal to zero. Wave patterns, depicting the normal component of the stress tensor, showed the reflected wave from the fracture and further spread of the seismic wave across the homogeneous medium.

Later on, we suppose to make the parallelization of the introduced algorithm for computing the seismic waves spread in a homogeneous medium with a fracture. It will help to speed up the computations significantly. In addition, the logic continuation of this work is the development of the analogous algorithm for computing fractured media for the $3 \mathrm{D}$ case.

\section{ACKNOWLedgments}

The reported study was funded by RFBR according to the research project № 19-01-00281.

\section{REFERENCES}

1. Muratov MV, Petrov IB. Application of Fractures Mathematical Models in Exploration Seismology Problems Modeling. Smart Innovation, Systems and Technologies, 2019. DOI: 10.1007/978-3-030-06228-6_11.

2. Bakulin A, Grechka V, Karaev N, Anisimov A, Kozlov E. Physical modeling and theoretical studies of seismic reflections from a fault zone. Society of Exploration Geophysicists Annual Meeting, 2004, 1674-1677.
3. Leviant VB, Miryakha VA, Muratov MV, Petrov IB. Seismic responses of vrtical fractures depending on their thickness. Tekbnologii seysmoraqvedki, 2015, 3:16-30 (in Russ.).

4. Leviant VB, Petrov IB, Muratov MV. Numerical simulation of wave responses from subvertical macrofractures system. Tekbnologii seysmorazvedki, 2012, 1:5-21 (in Russ.).

5. Carcione J. Scattering of elastic waves by a plane crack of finite width in a transversely isotropic medium. Int.J. Numer. Anal. Meth. Geomech., 1998, 22(4):263-275.

6. Hudson, JA. Overall properties of a cracked solid. Mathematical Proceedings of Cambridge Philosophical Society, 1980, 88:371-384.

7. Schoenberg M. Elastic wave behavior across linear slip interfaces. The Journal of the Acoustical Society of America, 1980, 68(5):1516-1521.

8. Grechka V, Kachanov M. Effective elasticity of fractured rocks: A snapshot of the work in progress. Geophysics, 2006, 71:W95-W58.

9. Qiwei Zhan, Qingtao Sun, Qiang Ren, Yuan Fang, Hua Wang, Qing Huo Liu. A discontinuous Galerkin method for simulating the effects of arbitrary discrete fractures on elastic wave propagation. Geophysical Journal International. 2017, 210(2):1219-1230.

10. Coates RT, Shoenberg M. Finite-difference modeling of faults and fractures. Geophysics, 1995, 60:1514-1523.

11. Zhan Q, Sun Q, Zhuang M, Mao Y, Ren Q, Fang Y, Huang W, Liu Q. A new upwind flux for a jump boundary condition applied to $3 \mathrm{D}$ viscous fracture modeling. Comput. Methods Appl. Mech. Eng., 2018, 331:456-473.

12. Novatskii VK. Teoriya uprugosti [Elasticity theory]. Moscow, Mir Publ., 1975.

13. Hsu CJ, Schoenberg M. Elastic waves through a simulated fractured medium. Geophysics, 1993, 58(7):964-977.

14. Liu E, Hudson JA, Pointer T. Equivalent medium representation of fractured rock. J. geophys. Res., 2000, 105:2981-3000.

15. Zhang J. Elastic wave modeling in fractured media with an explicit approach. Geophysics, 2005., 70:75-T85. 
16. Magomedov KM, Kholodov AS. Setochnokharakteristicheskie chislennye metody [Gridcharacteristic numerical methods]. Moscow, Nauka Publ., 1988.

17. Petrov IB, Lobanov AI. Lektsii po vychislitelnoy matematike [Computational mathematics lectures]. Moscow, Internet-University of Informational Technologies Publ., 2006.

18. LeVeque R. Finite-Volume Methods for Hyperbolic Problems. Cambridge University Press, 2002.

19. Petrov D. Application of grid-characteristic method to some seismic exploration problems in the Arctic. Journal of Physics Conference Series, 955(1):012029.

20. Ivanov A, Khokhlov N. Efficient inter-process communication in parallel implementation of grid-characteristic method. Smart Innovation, Systems and Technologies, 2019. DOI: 10.1007/978-3-030-06228-6_9. 